\title{
Selection of Optimum Nonlinear Confusion Component of Information Confidentiality Mechanism Using Grey Theory Based Decision-Making Technique
}

\author{
Nabilah Abughazalah ${ }^{a}$, Majid Khan ${ }^{b, 1}$ \\ ${ }^{a}$ Department of Mathematical Sciences, College of Science, Princess Nourah bint Abdulrahman \\ University, P.O.Box 84428,Riyadh 11671, Saudi Arabia \\ ${ }^{b}$ Department of Applied Mathematics and Statistics, Institute of Space Technology, Islamabad Pakistan
}

\begin{abstract}
In this age of internet communication, the security of digital information is one of the main issues. The privacy of data depends upon the encryption using some secure algorithm. The selection of robust cryptosystems to ensure confidentiality is a major concern to decrease the risk of cryptographic attacks. In this article, we have implemented a grey theory-based decision-making technique for the election of a robust cryptosystem that complies with all the cryptographic parameters. Six different already proposed encryption algorithms are selected as the alternatives of the decision-making problem and the parameters concerned for the decision are entropy, correlation coefficient, the number of pixels changing rate (NPCR), unified average changing intensity (UACI). The algorithm ranked as first by using grey-based decision-making method can be utilized for secure data encryption.
\end{abstract}

Keywords: Grey theory, Grey set, MCDM, Decision making problem, Cryptosystem, Encryption algorithm.

\section{Introduction}

In the current phase of digital media, the fast spread of confidential data by using the internet is usual. In the arena of information security, with the widespread communication ways, the security of multimedia devices becomes a major issue. Cryptographic means are the best possibility to secure the internet data by some encryption process. The information transferred by some insecure channels of communication can be protected by implementing some secure encryption algorithm. There exist many encryption algorithms in the literature that claim to provide confidentiality.

Cryptographic algorithms are divided into two major branches concerning the private key of the algorithm; the first one is the symmetric algorithms in which the encryption and decryption are done by using the same key; the second one is the asymmetric algorithms in which private key for encryption and decryption is different. Many conventional symmetric encryption systems are applied as a standard to secure communication such as Data Encryption Standard (DES) [1], Triple Data Encryption Standard (TDES) [2], and Advanced Encryption Standard (AES) [3]. Some of these structures are easy to attack by some cryptanalyst. The security of encryption structures depends on the private key of the algorithm. There are also many techniques proposed by researchers which support the requirements of secure encryption structures [4-15]. Unfortunately, all the offered encryption approaches cannot impart robustness. Recently, numerous encryption structures were attacked by cryptanalysts due to the weak implementation of private keys [16-20].

${ }^{1}$ Corresponding author: mk.cfd1 @gmail.com 
The major issue in the implementation of an encryption algorithm is the choice of a secure algorithm. Due to the presence of many encryption algorithms the choice of the best algorithm is difficult to reduce the risk of information leakage.

Multiple criteria decision making (MCDM) is a means for handling complex problems of engineering and science with incomplete and uncertain information. Many decision-making approaches are utilized in real-world problems [21-32,37,38]. There are numerous MCDM techniques, and each approach has its limitation according to the known information of the problem. Generally, decision-making approaches are executed on the problems with uncertain information in which the selection of the best alternative is hard by using some numerical features. Grey theory is one of the decision-making procedures utilized for the selection of best selection of alternatives by the means of uncertain information [33]. The grey theory also links with the conditions of fuzziness under some specific conditions [34-36].

In this paper, we have discussed the grey decision-making method for the election of a robust cryptosystem. We have selected some already existing encryption techniques which can be utilized for secure communication. As the selection of a secure encryption structure is a tough choice due to some uncertainty in the cryptographic properties of the algorithm. Some encryption algorithm successfully achieves the idealness of one property but may fail to achieve the standard in other features. Therefore, manual selection of cryptosystems to provide the best security is a very hard criterion. The grey theory is executed on some statistical properties of cryptosystems which are entropy, correlation coefficient, NPCR, and UACI. These parameters are considered as the attributes and six cryptosystems are taken as alternatives to the problem. The algorithm ranked as first by using grey theory-based decision-making method is declared as a secure and robust encryption system.

The persisting manuscript is arranged as follows: Section 2 offers some elementary definitions; In section 3 we have presented the statistical properties of some understudy cryptosystems; section 4 offers the basic idea of grey theory-based decision-making problem; in the next section, we have implemented grey theory for the selection of robust cryptosystem; finally, the conclusion is drawn.

\section{Some Basic Preliminaries}

Grey theory was initially proposed in 1982 by Deng [33]. This theory leads towards the solution of uncertainty problems with incomplete information and discrete data by using grey sets. In this segment, we have described some fundamental definitions related to the grey theory.

\section{Definition: 2.1}

Consider $U$ be the universal set consisting of all possible alternatives. The grey set $C$ over $U$ is defined by two mappings $\bar{\gamma}_{C}(u)$ and $\underline{\gamma}_{C}(u)$. Mathematically, mapping can be defined as:

$$
\left\{\begin{array}{l}
\bar{\gamma}_{C}(u): u \rightarrow[0,1] \\
\underline{\gamma}_{C}(u): u \rightarrow[0,1] .
\end{array}\right.
$$

With the condition $\bar{\gamma}_{C}(u) \geq \underline{\gamma}_{C}(u)$ for all $u \in U, \bar{\gamma}_{C}(u)$ and $\underline{\gamma}_{C}(u)$ are the upper and lower membership functions in $C$ respectively. 


\section{Definition: 2.2}

The grey set $C$ over the universal set $U$ turn out to be a fuzzy set if the upper and lower membership functions are the same. Mathematically it can be written as $\bar{\gamma}_{C}(u)=\underline{\gamma}_{C}(u)$. It indicates that grey theory can also link with the condition of fuzziness.

\section{Definition: 2.3}

A number with uncertain information can be indicated as the grey number. For example, if the attributes ratings are defined in some linguistic variable; there with an interval stating it numerically. This interval will encompass vague data. Usually, the grey number is written as $\otimes C=\left.C\right|_{\underline{\gamma}} ^{\bar{\gamma}}$.

\section{Definition: 2.4}

The grey set $C$ can be described as the grey number with a lower limit and upper limit by $\otimes C=[\underline{C}, \infty)$ and $\otimes C=(\infty, \bar{C})$ respectively.

\section{Definition: 2.5}

The grey set with the upper and lower limits can be defined as an interval grey number by $\otimes C=[\underline{C}, \bar{C}]$

\section{Definition: 2.6 [37]}

R. E, Moore's developed the interval operations [38]. Grey number operation is specified on the set of intervals, instead of real numbers. The fundamental operations for two grey numbers $\otimes C_{1}=\left[\underline{C}_{1}, \bar{C}_{1}\right]$ and $\otimes C_{2}=\left[\underline{C}_{2}, \bar{C}_{2}\right]$ can be defined mathematically as:

$$
\begin{gathered}
\otimes C_{1}+\otimes C_{2}=\left[\underline{C}_{1}+\underline{C}_{2}, \bar{C}_{1}+\bar{C}_{2}\right], \\
\otimes C_{1}-\otimes C_{2}=\left[\underline{C}_{1}-\underline{C}_{2}, \bar{C}_{1}-\bar{C}_{2}\right], \\
\otimes C_{1} \times \otimes C_{2}=\left[\min \left(\underline{C}_{1} \underline{C}_{2}, \underline{C}_{1} \bar{C}_{2}, \bar{C}_{1} \underline{C}_{2}, \bar{C}_{1} \bar{C}_{2}\right), \max \left(\underline{C}_{1} \underline{C}_{2}, \underline{C}_{1} \bar{C}_{2}, \bar{C}_{1} \underline{C}_{2}, \bar{C}_{1} \bar{C}_{2}\right)\right], \\
\otimes C_{1} \div \otimes C_{2}=\left[\underline{C}_{1}, \bar{C}_{1}\right] \times\left[\frac{1}{\underline{C}_{2}}, \frac{1}{\bar{C}_{2}}\right] .
\end{gathered}
$$

\section{Definition: 2.7}

The length of the upper and lower limit of a grey number $\otimes C=[\underline{C}, \bar{C}]$ can be calculated by 


$$
L(\otimes C)=[\bar{C}-\underline{C}]
$$

\section{Definition: 2.8 [37]}

The possibility degree $\otimes C_{1} \leq \otimes C_{2}$ for two grey numbers $\otimes C_{1}=\left[\underline{C}_{1}, \bar{C}_{1}\right]$ and $\otimes C_{2}=\left[\underline{C}_{2}, \bar{C}_{2}\right]$ can be determined by the following mathematical expression:

$$
P\left\{\otimes C_{1} \leq \otimes C_{2}\right\}=\frac{\max \left(0, L^{*}-\max \left(0, \bar{C}_{1}-\underline{C}_{2}\right)\right)}{L^{*}},
$$

Where $L^{*}=L\left(\otimes C_{1}\right)+L\left(\otimes C_{2}\right)$.

The possibility degree lies on the real axis in the following four conditions:

1. If $\bar{C}_{1}=\bar{C}_{2}$ and $\underline{C}_{1}=\underline{C}_{2}$, or in other words, we can say that $\otimes C_{1}$ and $\otimes C_{2}$ are equal. Then possibility degree $P\left\{\otimes C_{1} \leq \otimes C_{2}\right\}=0.5$.

2. If $\bar{C}_{2}<\underline{C}_{1}$, we can say that $\otimes C_{2}$ is smaller than $\otimes C_{1}$. Then $P\left\{\otimes C_{1} \leq \otimes C_{2}\right\}=0$.

3. If $\underline{C}_{2}<\bar{C}_{1}$, we say that $\otimes C_{2}$ is greater than $\otimes C_{1}$. Then $P\left\{\otimes C_{1} \leq \otimes C_{2}\right\}=1$.

4. If $P\left\{\otimes C_{1} \leq \otimes C_{2}\right\}>0.5$ we can say that $\otimes C_{2}>\otimes C_{1}$ and if $P\left\{\otimes C_{1} \leq \otimes C_{2}\right\}<0.5$ we can say

\begin{tabular}{|c|c|c|c|}
\hline \multicolumn{2}{|c|}{ Attribute weights } & \multicolumn{2}{|c|}{ Attribute rating } \\
\hline Linguistic variable & $\otimes \omega$ & Linguistic variable & $\otimes C$ \\
\hline Very less (VL) & {$[0.0,0.1]$} & Very bad (VB) & {$[0,1]$} \\
\hline Less (L) & {$[0.1,0.3]$} & $\mathrm{Bad}(\mathrm{B})$ & {$[1,3]$} \\
\hline Average less (AL) & {$[0.3,0.4]$} & Average bad (AB) & {$[3,4]$} \\
\hline Average (A) & {$[0.4,0.5]$} & Fair $(F)$ & {$[4,5]$} \\
\hline Average high $(\mathrm{AH})$ & {$[0.5,0.6]$} & Average good (AG) & {$[5,6]$} \\
\hline $\operatorname{High}(\mathrm{H})$ & {$[0.6,0.9]$} & $\operatorname{Good}(\mathrm{G})$ & {$[6,9]$} \\
\hline Very high $(\mathrm{VH})$ & {$[0.9,1.0]$} & Very good (VG) & {$[9,10]$} \\
\hline
\end{tabular}
that $\otimes C_{2}<\otimes C_{1}$.

\section{Understudy Cryptosystems and their Statistical Properties}

Many cryptographic algorithms claim to be secure for data communication. But all of them do not provide confidentiality. In this segment, we have selected some cryptosystems for the selection of robust algorithms through the grey-based decision-making approach. In Ref. [10] construct encryption procedure built on a chaotic map, elliptic curves, and Substitution-box. Authors in Ref. [11] proposed a cryptosystem based on generalized chaotic maps and fractional-order edge detection. J. B. Lima et al. [12] offered a medical image encryption algorithm by using cosine number transform. In [13] authors presented an improved Elgamal based encryption technique. X. In [14] authors developed an elliptic curves-based chaotic encryption scheme. Zhang and X. Wang [15] proposed a novel cryptosystem based on elliptic curves. 


\begin{tabular}{lcccc}
\hline Scheme & Entropy & Correlation & NPCR & UACI \\
\hline S. Ibrahim et. al. [10] & 7.9994 & 0.0088 & 99.6086 & 33.4409 \\
S. M. Ismail et al. [11] & 7.9972 & -0.0001 & 76.1681 & 24.2534 \\
J. B. Lima et al. [12] & 7.9992 & -0.0007 & 99.6082 & 33.4682 \\
J. B. Laip. Et al. [13] & 7.9994 & 0.0024 & 99.6071 & 33.4423 \\
A. M. Abbas et al. [14] & 7.9998 & -0.0006 & 99.6095 & 33.4621 \\
X. Zhang et al. [15] & 7.9986 & 0.0034 & 99.9500 & 33.1100 \\
\hline
\end{tabular}

The evaluation of the security of cryptographic algorithms is carried out by some statistical analysis. The statistical analyses considered for the evaluation of the cryptosystems are, entropy, correlation coefficient, NPCR, and UACI [9]. We have listed the values of some statistical analysis of understudy cryptosystems in Table 2 . The bar chart illustration of statistical properties of understudy cryptosystems is shown in Fig. 1.

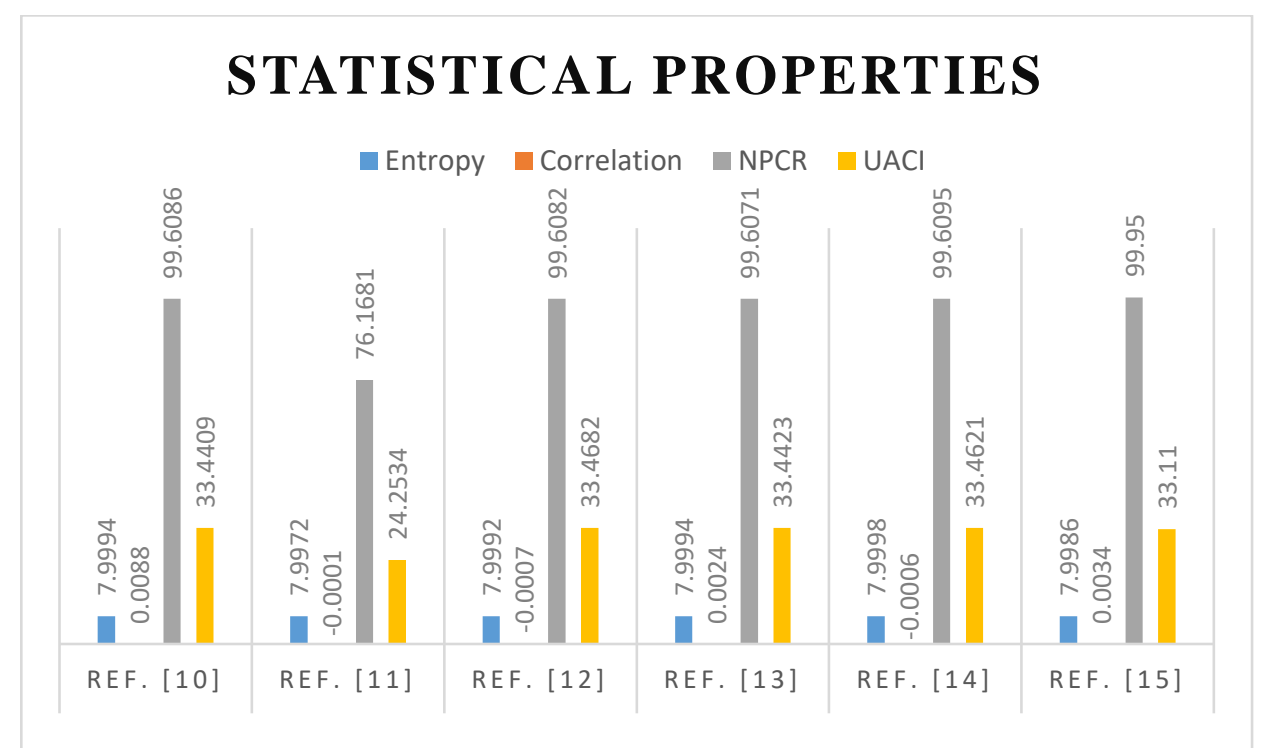

Fig. 1: Bar chart representation of statistical properties of understudy cryptosystems.

\section{Grey Theory Based Decision-Making Method [33]}

The grey theory-based decision-making method is for ordering the preferences of any alternatives. This approach is very useful for solving problems with uncertain information. Consider that $C=\left\{C_{1}, C_{2}, \ldots, C_{n}\right\}$ be the set of all possible options and $A=\left\{A_{1}, A_{2}, \ldots, A_{m}\right\}$ be the set of all possible parameters of the problem. The weight vector for attributes is taken as $\otimes \omega=\left\{\otimes \omega_{1}, \otimes \omega_{2}, \ldots, \otimes \omega_{m}\right\}$. In this approach, the weight and ratings of the attributes are taken as linguistic variables. The numerical scale for attribute weight and attribute rating is defined in Table 1. The grey decision-making approach can be reviewed by the subsequent steps:

Step 1: Firstly, the weight of each attribute is assigned by some decision-makers in the linguistic variable. Suppose that there are $Q$ decision-makers who make the decision in linguistic variable and the resultant weight is calculated by converting them into the numerical scale by using the results in Table 1 . The weight for attributes is selected as: 


$$
\otimes \omega_{q}=\frac{1}{Q}\left[\otimes \omega_{q}^{1}+\otimes \omega_{q}^{2}+\ldots+\otimes \omega_{q}^{Q}\right]
$$

Where $\otimes \omega_{q}^{Q},(q=1,2, \ldots, m)$ is the attribute weight of the $Q$ th decision-maker and can be stated in the structure of grey numbers as $\otimes \omega_{q}^{Q}=\left[\underline{\omega}_{q}^{Q}, \bar{\omega}_{q}^{Q}\right]$.

Step 2: The second step is the rating of the attribute by $Q$ decision-makers in linguistic variables which are converted into numerical data by using Table 1 . The rating is for each attribute is calculated by:

$$
\otimes C_{p q}=\frac{1}{Q}\left[\otimes C_{p q}^{1}+C_{p q}^{2}+\ldots+C_{p q}^{Q}\right],
$$

Where $\otimes C_{p q}^{Q},(p=1,2, \ldots, n ; q=1,2, \ldots, m)$ denotes the rating attribute of $Q$ th decision-maker and can be stated in the form of grey numbers as $\otimes C_{p q}^{Q}=\left[\underline{C}_{p q}^{Q}, \bar{C}_{p q}^{Q}\right]$.

Step 3: Next, the grey decision matrix (GDM) is constructed based on the grey attribute rating obtained in the previous step as follows:

$$
G D M=\left[\begin{array}{cccc}
\otimes C_{11} & \otimes C_{12} & \cdots & \otimes C_{1 m} \\
\otimes C_{21} & \otimes C_{22} & \cdots & \otimes C_{2 m} \\
\vdots & \vdots & \ddots & \vdots \\
\otimes C_{n 1} & \otimes C_{n 2} & \cdots & \otimes C_{n m}
\end{array}\right]
$$

where $\otimes C_{p q}$ denotes the grey rating attributes.

Step 4: In this step, the grey decision matric (GDM) obtained from the previous step is normalized and defined mathematically as:

$$
\text { Normalized_GDM=[ }\left[\begin{array}{cccc}
\otimes C_{11}^{*} & \otimes C_{12}^{*} & \cdots & \otimes C_{1 m}^{*} \\
\otimes C_{21}^{*} & \otimes C_{22}^{*} & \cdots & \otimes C_{2 m}^{*} \\
\vdots & \vdots & \ddots & \vdots \\
\otimes C_{n 1}^{*} & \otimes C_{n 2}^{*} & \cdots & \otimes C_{n m}^{*}
\end{array}\right],
$$

where for beneficial attribute, $\otimes C_{p q}$ is defined as:

$$
\otimes C_{p q}^{*}=\left[\frac{\underline{C}_{p q}}{C_{q}^{\max }}, \frac{\bar{C}_{p q}}{C_{q}^{\max }}\right],
$$

For $C_{q}^{\max }=\max _{1 \leq p \leq n}\left(\left\{\bar{C}_{p q}\right\}\right)$. And for non-beneficial attributes $\otimes C_{p q}$ is defined as: 


$$
\otimes C_{p q}^{*}=\left[\frac{C_{q}^{\min }}{\bar{C}_{p q}}, \frac{C_{q}^{\min }}{\underline{C}_{p q}}\right],
$$

For $C_{q}^{\min }=\min _{1 \leq p \leq n}\left(\left\{\underline{C}_{p q}\right\}\right)$.

Step 5: Further, the weight vector is multiplied with normalized GDM to obtain the weighted normalized GDM by:

$$
\text { Weighted_normalized_GDM }^{*}=\left[\begin{array}{cccc}
\otimes R_{11} & \otimes R_{12} & \cdots & \otimes R_{1 m} \\
\otimes R_{21} & \otimes R_{22} & \cdots & \otimes R_{2 m} \\
\vdots & \vdots & \ddots & \vdots \\
\otimes R_{n 1} & \otimes R_{n 2} & \cdots & \otimes R_{n m}
\end{array}\right]
$$

where $\otimes R_{p q}=\otimes C_{p q}^{*} \times \omega_{q}$.

Step 6: The ideal referential alternatives are selected from weighted normalized GDM acquired in step 5. The mathematical expression for ideal referential alternatives is defined as:

$$
I^{\max }=\left\{\left[\max _{1 \leq p \leq n} \underline{R}_{p 1}, \max _{1 \leq p \leq n} \bar{R}_{p 1}\right],\left[\max _{1 \leq p \leq n} \underline{R}_{p 2}, \max _{1 \leq p \leq n} \bar{R}_{p 2}\right], \ldots,\left[\max _{1 \leq p \leq n} \underline{R}_{p m}, \max _{1 \leq p \leq n} \bar{R}_{p m}\right]\right\}
$$

Step 7: This step involves the calculation of the grey possibility degree between the alternatives $S=\left\{C_{1}, C_{2}, \ldots, C_{m}\right\}$ and the ideal referential alternatives $I^{\max }$ by the following mathematical expression.

$$
P=\left\{S_{i} \leq I^{\max }\right\}=\frac{1}{n} \sum_{q=1}^{m} P\left\{\otimes R_{p q} \leq I_{q}^{\max }\right\}
$$

Step 8: The last step is the ranking of preference orders. If the grey possible degree is lesser then the ranking of choice is higher. Otherwise, in the case of greater grey possibility degree, the ranking order is worse.

\section{Application of Grey Theory-Based Decision-Making Method in the Selection of Robust Cryptosystem}

We have six possible cryptosystems as alternatives from which we need to select the robust one. The strength of cryptographic algorithms can be extinguished by the statistical properties entropy, correlation coefficient, NPCR, and UACI which are named as $A_{1}, A_{2}, A_{3}$ and $A_{4}$ respectively for grey-based decision-making method. All the possible cryptosystems are nominated as $C_{1}, C_{2}, C_{3}, C_{4}, C_{5}$, and $C_{6}$ . The selection of a strong cryptosystem is done by using the following steps:

Step 1: The first step is the calculation of the weight of the parameters $A_{1}, A_{2}, A_{3}$ and $A_{4}$ with the decision of four decision-makers $D M_{1}, D M_{2}, D M_{3}$, and $D M_{4}$. The weights of each parametric attribute 
are selected by employing the linguistic to the numeric scale defined in Table 1. By using Table 1 and Eq. (8) the calculated weight is defined in Table 3.

Table 3: Weight of each parameter according to each decision-maker.

\begin{tabular}{cccccc}
\hline$A_{i}$ & $D M_{1}$ & $D M_{2}$ & $D M_{3}$ & $D M_{4}$ & $\otimes \omega_{q}$ \\
\hline$A_{1}$ & $\mathrm{AH}$ & $\mathrm{VH}$ & $\mathrm{H}$ & $\mathrm{VH}$ & {$[0.725,0.875]$} \\
$A_{2}$ & $\mathrm{~A}$ & $\mathrm{~A} \mathrm{H}$ & $\mathrm{A} \mathrm{H}$ & $\mathrm{H}$ & {$[0.5,0.65]$} \\
$A_{3}$ & $\mathrm{VH}$ & $\mathrm{H}$ & $\mathrm{VH}$ & $\mathrm{A} \mathrm{H}$ & {$[0.725,0.875]$} \\
$A_{4}$ & $\mathrm{VH}$ & $\mathrm{H}$ & $\mathrm{H}$ & $\mathrm{A} \mathrm{H}$ & {$[0.725,0.875]$} \\
\hline
\end{tabular}

Step 2: The next step is the calculation of attribute rating according to each decision-maker concerning Eq. (9) and the resultant ratings are depicted in Table 4.

Table 4: Rating of each alternative according to four decision-makers.

\begin{tabular}{|c|c|c|c|c|c|c|}
\hline$A_{i}$ & $C_{i}$ & $D M_{1}$ & $D M_{2}$ & $D M_{3}$ & $\mathrm{DM}_{4}$ & $\otimes \omega_{q}$ \\
\hline \multirow{6}{*}{$A_{1}$} & $C_{1}$ & $\mathrm{G}$ & VG & $\mathrm{G}$ & $\mathrm{AG}$ & {$[6.5,8.5]$} \\
\hline & $C_{2}$ & $\mathrm{~F}$ & $\mathrm{AG}$ & $\mathrm{AB}$ & $\mathrm{AG}$ & {$[4.25,5.25]$} \\
\hline & $C_{3}$ & G & G & $\mathrm{AG}$ & F & {$[5.25,7.25]$} \\
\hline & $C_{4}$ & G & VG & G & $\mathrm{AG}$ & {$[6.5,8.5]$} \\
\hline & $C_{5}$ & VG & VG & VG & $\mathrm{G}$ & {$[8.25,9.75]$} \\
\hline & $C_{6}$ & $\mathrm{AG}$ & $\mathrm{AG}$ & $\mathrm{F}$ & $\mathrm{F}$ & {$[4.5,5.5]$} \\
\hline \multirow{9}{*}{$A_{2}$} & $C_{1}$ & $\mathrm{~F}$ & $\mathrm{AG}$ & $\mathrm{AB}$ & F & {$[4,5]$} \\
\hline & $C_{2}$ & VG & VG & G & $\mathrm{G}$ & {$[7.5,9.5]$} \\
\hline & $C_{3}$ & VG & VG & VG & VG & {$[9,10]$} \\
\hline & $C_{4}$ & $\mathrm{AG}$ & G & $\mathrm{F}$ & $\mathrm{F}$ & {$[4.75,6.25]$} \\
\hline & $C_{5}$ & VG & VG & VG & VG & {$[9,10]$} \\
\hline & $C_{6}$ & $\mathrm{AG}$ & G & $\mathrm{F}$ & F & {$[4.75,6.25]$} \\
\hline & $C_{1}$ & G & $\mathrm{AG}$ & $\mathrm{AG}$ & G & {$[5.5,7.5]$} \\
\hline & $C_{2}$ & VB & B & B & B & {$[0.75,2.5]$} \\
\hline & $C_{3}$ & G & $\mathrm{AG}$ & $\mathrm{AG}$ & G & {$[5.5,7.5]$} \\
\hline
\end{tabular}




$\begin{array}{ccccccc}A_{3} & C_{4} & \mathrm{G} & \mathrm{AG} & \mathrm{F} & \mathrm{AG} & {[5,6.5]} \\ & C_{5} & \mathrm{G} & \mathrm{G} & \mathrm{G} & \mathrm{G} & {[6,9]} \\ & C_{6} & \mathrm{VG} & \mathrm{VG} & \mathrm{VG} & \mathrm{VG} & {[9,10]} \\ & & & \mathrm{GG} & \mathrm{AG} & \mathrm{AG} & {[5.25,6.75]} \\ & C_{1} & \mathrm{G} & \mathrm{AG} & \mathrm{V} & \mathrm{VB} & {[0.5,2]} \\ A_{4} & \mathrm{~B} & \mathrm{VB} & \mathrm{B} & \mathrm{VG} & {[8.25,9.75]} \\ & C_{2} & \mathrm{VG} & \mathrm{G} & \mathrm{VG} & \mathrm{AG} & {[5.5,7.5]} \\ & C_{3} & \mathrm{G} & \mathrm{AG} & \mathrm{G} & \mathrm{VG} & {[8.25,9.75]} \\ & C_{4} & \mathrm{VG} & \mathrm{G} & \mathrm{VG} & \mathrm{F} & {[4,5]} \\ & C_{5} & \mathrm{AG} & \mathrm{F} & \mathrm{AB} & \mathrm{F} & \end{array}$

Step 3: Next, the grey decision matrix (GDM) is created by applying the attribute ratings calculated in Table 4. The constructed GDM according to Eq. (10) is displayed in Table 5.

\begin{tabular}{lcccc}
\hline \multicolumn{5}{c}{ Table 5: Grey decision matrix for all possible alternatives. } \\
\hline$C_{i}$ & $A_{1}$ & $A_{2}$ & $A_{3}$ & $A_{4}$ \\
\hline$C_{1}$ & {$[6.5,8.5]$} & {$[4,5]$} & {$[5.5,7.5]$} & {$[5.25,6.75]$} \\
$C_{2}$ & {$[4.25,5.25]$} & {$[7.5,9.5]$} & {$[0.75,2.5]$} & {$[0.5,2]$} \\
$C_{3}$ & {$[5.25,7.25]$} & {$[9,10]$} & {$[5.5,7.5]$} & {$[8.25,9.75]$} \\
$C_{4}$ & {$[6.5,8.5]$} & {$[4.75,6.25]$} & {$[5,6.5]$} & {$[5.5,7.5]$} \\
$C_{5}$ & {$[8.25,9.75]$} & {$[9,10]$} & {$[6,9]$} & {$[8.25,9.75]$} \\
$C_{6}$ & {$[4.5,5.5]$} & {$[4.75,6.25]$} & {$[9,10]$} & {$[4,5]$} \\
\hline
\end{tabular}

Step 4: In this step, the normalization of GDM is carried out by using Eq. (11)-(13). For the process of normalization, the entropy, NPCR, and UACI are considered beneficial attributes, and correlation is taken as a non-beneficial attribute. The normalized GDM is demonstrated in Table 6.

\begin{tabular}{lcccc}
\hline \multicolumn{5}{c}{ Table 6: Normalized grey decision matrix. } \\
\hline$C_{i}$ & $A_{1}$ & $A_{2}$ & $A_{3}$ & $A_{4}$ \\
\hline$C_{1}$ & {$[0.667,0.872]$} & {$[0.400,0.500]$} & {$[0.550,0.750]$} & {$[0.074,0.095]$} \\
$C_{2}$ & {$[0.436,0.538]$} & {$[0.750,0.950]$} & {$[0.075,0.250]$} & {$[0.250,1.000]$} \\
$C_{3}$ & {$[0.538,0.744]$} & {$[0.900,1.000]$} & {$[0.550,0.750]$} & {$[0.051,0.061]$} \\
$C_{4}$ & {$[0.667,0.872]$} & {$[0.475,0.625]$} & {$[0.500,0.650]$} & {$[0.067,0.091]$} \\
$C_{5}$ & {$[0.841,1.000]$} & {$[0.900,1.000]$} & {$[0.600,0.900]$} & {$[0.051,0.061]$} \\
$C_{6}$ & {$[0.462,0.564]$} & {$[0.475,0.625]$} & {$[0.900,1.000]$} & {$[0.100,0.125]$} \\
\hline
\end{tabular}


Step 5: After the calculation of normalized GDM, the weight vectors are multiplied with it to get weighted normalized GDM. The weight vectors calculated in Table 3 are multiplied with the normalized vectors in Table 5 by using the mathematical expression defined in Eq. (14). The calculated weighted normalized GDM is presented in Table 7.

\begin{tabular}{lcccc}
\hline \multicolumn{5}{c}{ Table 7: Weighted normalized GDM. } \\
\hline$C_{i}$ & $A_{1}$ & $A_{2}$ & $A_{3}$ & $A_{4}$ \\
\hline$C_{1}$ & {$[0.483,0.763]$} & {$[0.200,0.325]$} & {$[0.399,0.656]$} & {$[0.054,0.083]$} \\
$C_{2}$ & {$[0.316,0.471]$} & {$[0.375,0.618]$} & {$[0.054,0.219]$} & {$[0.181,0.875]$} \\
$C_{3}$ & {$[0.390,0.651]$} & {$[0.450,0.650]$} & {$[0.399,0.656]$} & {$[0.037,0.053]$} \\
$C_{4}$ & {$[0.483,0.763]$} & {$[0.238,0.406]$} & {$[0.363,0.569]$} & {$[0.048,0.080]$} \\
$C_{5}$ & {$[0.610,0.875]$} & {$[0.450,0.650]$} & {$[0.435,0.788]$} & {$[0.037,0.053]$} \\
$C_{6}$ & {$[0.335,0.494]$} & {$[0.238,0.406]$} & {$[0.653,0.875]$} & {$[0.073,0.109]$} \\
\hline
\end{tabular}

Step 6: The ideal referential alternatives $I^{\max }$ are selected from weighted normalized GDM acquired in step 5 . The selection of ideal referential alternatives is done by using the mathematical expression defined in Eq. (15) as:

$$
I^{\max }=\{[0.610,0.875],[0.450,0.650],[0.653,0.875],[0.181,0.875]\} .
$$

Step 7: In this step, the gray possibility degree is calculated between all the six alternatives $C_{i}$ and the ideal referential alternative $I^{\max }$. Corresponding to Eq. (15), the possibility degree for each alternative is calculated as

$$
\begin{gathered}
P\left(C_{1} \leq I^{\max }\right)=0.928, \\
P\left(C_{2} \leq I^{\max }\right)=0.780, \\
P\left(C_{3} \leq I^{\max }\right)=0.854, \\
P\left(C_{4} \leq I^{\max }\right)=0.930, \\
P\left(C_{5} \leq I^{\max }\right)=0.691, \\
P\left(C_{6} \leq I^{\max }\right)=0.875 .
\end{gathered}
$$

Step 7: Ranking of liking order is done according to the possibility degree. If the rate of possibility degree is lowest the rank of preference order is highest. According to the possibility degree calculated in the previous step the ranking of all possible alternatives can be done as: 


$$
C_{5}>C_{2}>C_{3}>C_{6}>C_{1}>C_{4}
$$

From the ranking of preference order, we can say that the cryptosystem $C_{5}[14]$ is the best and robust among all the possible cryptosystem inserted in the grey decision-making approach. The bar chart representation of the ranking of each alternative is depicted in Fig. 2 which reflects that the cryptosystem $C_{5}$ is best among all the alternatives.

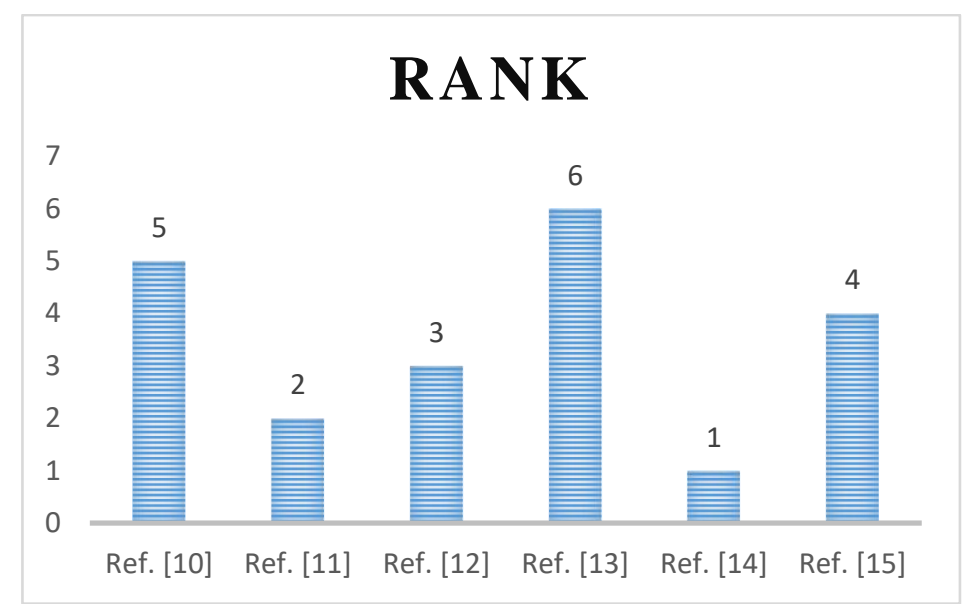

Fig. 2: Bar chart representation of ranking of each alternative.

\section{Conclusion}

In this work, we have implemented a grey theory-based decision-making method for the election of robust cryptosystem. The grey theory is executed on six cryptosystems considered as all possible alternatives of the problem. The parameter utilized for the selection procedure is entropy, correlation coefficient, NPCR, and UACI. The grey theory-based approach uses some decision-makers for the best selection. In this work, we have involved four decision-makers who decide the priority of each parameter. The first ranked algorithm by using the grey approach is the cryptosystem proposed by A. M. Abbas [14] which depends on elliptic curves. Therefore, we can conclude that the cryptosystem offered in [14] can be used for secure communication and the grey theory-based decision-making method can be successfully implemented for the selection of cryptographic problems.

\section{Acknowledgment}

This research was funded by Princess Nourah bint Abdulrahman University Researchers Supporting Project Number (PNURSP2022R87), Princess Nourah bint Abdulrahman University, Riyadh, Saudi Arabia.

\section{Funding}

This research was funded by Princess Nourah bint Abdulrahman University Researchers Supporting Project Number (PNURSP2022R87), Princess Nourah bint Abdulrahman University, Riyadh, Saudi Arabia.

Conflicts of interest: The authors declare that they have no conflict of interest.

Ethical Approval: This article does not contain any studies with human participants or animals performed by any of the authors.

Data Availability Statement: The authors declare that data supporting the findings of this study are available within the article. 


\section{References}

[1]. Walter Tuchman (1997). "A brief history of the data encryption standard". Internet besieged: countering cyberspace scofflaws. ACM Press/Addison-Wesley Publishing Co. New York, NY, USA. pp. 275-280.

[2]. "Triple DES Encryption". IBM. Retrieved 2010-05-17.

[3]. "Advanced Encryption Standard (AES)" (PDF). Federal Information Processing Standards. 26 November 2001. doi:10.6028/NIST.FIPS.197. 197.

[4]. Munir N, Khan M. A Generalization of Algebraic Expression for Nonlinear Component of Symmetric Key Algorithms of Any Characteristic p. ICAEM 2018-2018 International Conference on Applied and Engineering Mathematics, Proceedings. 2018. pp. 48-52.

[5]. Alghafis, A., Munir, N., Khan, M. et al. An Encryption Scheme Based on Discrete Quantum Map and Continuous Chaotic System. Int J Theor Phys (2020).

[6]. Khan M, Munir N (2019) A novel image encryption technique based on generalized advanced encryption standard based on field of any characteristic, Wireless Pers Commun 109:849-867. https://doi.org/10.1007/s11277-019-06594-6.

[7]. Cheng G, Wang C, Chen H (2019) A novel color image encryption algorithm based on hyper chaotic system and permutation-diffusion architecture. Int J Bifurcation Chaos 29(09):1950115

[8]. Alghafis, A., Munir, N. \& Khan, M. An encryption scheme based on chaotic Rabinovich-Fabrikant system and S8 confusion component. Multimed Tools Appl (2020). https://doi.org10.1007s11042020-10142-X

[9]. Munir N, Khan M, Wei Z, Akgul A, Amin M, Hussain I (2020) Circuit implementation of 3D chaotic selfexciting single-disk homopolar dynamo and its application in digital image confidentiality. Wireless Netw. https://doi.org/10.1007/s11276-020-02361-9.

[10]. S. Ibrahim and A. Alharbi, “Efficient image encryption scheme using Henon map, dynamic Sboxes and elliptic curve cryptography," IEEE Access, vol. 8, pp. 194289_194302, 2020.

[11]. S. M. Ismail, L. A. Said, A. G. Radwan, A. H. Madian, and M. F. Abu-ElYazeed, “'A novel image encryption system merging fractional-order edge detection and generalized chaotic maps," Signal Process., vol. 167, Feb. 2020, Art. no. 107280.

[12]. J. B. Lima, F. Madeiro, and F. J. R. Sales, “'Encryption of medical images based on the cosine number transform," Signal Process., Image Commun., vol. 35, pp. 1_8, Jul. 2015.

[13]. D. S. Laiphrakpam and M. S. Khumanthem, "'Medical image encryption based on improved ElGamal encryption technique," Optik, vol. 147, pp. 88_102, Oct. 2017.

[14]. A. M. Abbas, A. A. Alharbi and S. Ibrahim, "A Novel Parallelizable Chaotic Image Encryption Scheme Based on Elliptic Curves," in IEEE Access, vol. 9, pp. 54978-54991, 2021, doi: 10.1109/ACCESS.2021.3068931.

[15]. X. Zhang and X. Wang, "'Digital image encryption algorithm based on elliptic curve public cryptosystem," IEEE Access, vol. 6, pp. 70025_70034, 2018.

[16]. Dhall S, Pal SK, Sharma K (2017) Cryptanalysis of image encryption based on a new 1D chaotic system, Signal processing, PII S0165-1684(17)30434-6, https://doi.org/10.1016/j.sigpro.2017.12.021.

[17]. Zhang Y, Xiao D, Wen W, Nan H (2014) Cryptanalysis of image scrambling based on chaotic sequences and vigen'ere cipher. Nonlinear Dynamics 78(1):235-240. 
[18]. Ahmad M, Al Solami E, Wang X-Y, Doja MN, Beg MMS, Alzaidi AA (2018) Cryptanalysis of an image encryption algorithm based on combined chaos for BAN system and its improved scheme using SHA-512 and hyperchaos. Symmetry 10(7):266.

[19]. Noor Munir, Majid Khan, Tariq Shah, Ammar S. Alanazi, Iqtadar Hussain, Cryptanalysis of nonlinear confusion component based encryption algorithm, Integration, Volume 79,2021, Pages 41-47,ISSN 0167-9260.

[20]. C. Li, S. Li, G. Chen, G. Chen, L. Hu, Cryptanalysis of a new signal security system for multimedia data transmission, EURASIP Journal on Applied Signal Processing 2005 (8) (2005) 1277-1288.

[21]. J. Choi, S.X. Bai, J. Geunes, H.E. Romeijn, Manufacturing delivery performance for supply chain management, Mathematical and Computer Modelling 45 (2007) 11-20.

[22]. W.D. Hong, B. Lyes, X.L. Xie, A simulation optimization methodology for supplier selection problem, International Journal of Computer Integrated Manufacturing 18 (2-3) (2005) 210-224.

[23]. N.O. Ndubisi, M. Jantan, L.C. King, M.S. Ayub, Supplier selection and management strategies and manufacturing flexibility, Journal of Enterprise Information Management 18 (3) (2005) 330349.

[24]. R. Lasch, C.G. Janker, Supplier selection and controlling using multivariate analysis, International Journal of Physical Distribution and Logistics Management 35 (6) (2005) 409-425.

[25]. K.N. Thompson, Vendor profile analysis, Journal of Purchasing and Materials Management 26 (1) (1990) 11-18.

[26]. E. Timmerman, An approach to vendor performance evaluation, Journal of Purchasing and Supply Management 1 (1986) 27-32.

[27]. G. Barbarosoglu, T. Yazgac, An application of the analytic hierarchy process to the supplier selection problem, Production and Inventory Management Journal 1st quarter (1997) 14-21.

[28]. R. Narasimhan, An analytic approach to supplier selection, Journal of Purchasing and Supply Management 1 (1983) 27-32.

[29]. J. Sarkis, S. Talluri, A model for strategic supplier selection, in: Ninth Int Conf on IPSERA, 2000, pp. 652-661.

[30]. R.M. Monezka, S.J. Trecha, Cost-based supplier performance evaluation, Journal of Purchasing and Materials Management 24 (2) (1998) 2-7.

[31]. D.L. Smytka, M.W. Clemens, Total cost supplier selection model: A case study, International Journal of Purchasing and Materials Management 29 (1) (1993) 42-49.

[32]. F.P. Buffa,W.M. Jackson, A goal programming model for purchase planning, Journal of Purchasing and Materials Management 19 (3) (1983) 27-34.

[33]. J.L. Deng, The introduction of grey system, The Journal of Grey System 1 (1) (1989) 1-24.

[34]. Y.X. Wang, Application of fuzzy decision optimum model in selecting supplier, The Journal of Science Technology and Engineering 5 (15) (2005) 1100-1103.

[35]. L.A. Zadeh, Fuzzy sets, Information and Control 8 (1965) 338-353.

[36]. R.E. Bellman, L.A. Zadeh, Decision-making in a fuzzy environment, Management Science 17 (4) (1970) 141-164.

[37]. J.R. Shi, S.Y. Liu, W.T. Xiong, A new solution for interval number linear programming, Journal of Systems Engineering Theory and Practice 2 (2005) 101-106.

[38]. R.E. Moore, Interval Analysis, Prentice-Hall, Englewood Cliffs, NJ, 1966. 